\title{
A comparative study between different types of local flaps used for soft tissue reconstruction of volar thumb defects
}

\begin{abstract}
Management of soft tissue defects of the thumb finger represents challenge for plastic surgeons regarding techniques, cosmetic and functional results.
\end{abstract}

\begin{abstract}
Aim of study: The present study was conducted to evaluate a comparison between three types of local flaps used for reconstructing soft tissue defects of the volar aspect of the thumb, volar palmar advancement flap, heterodigital neurovascular island flap and first dorsal metacarpal artery flap.
\end{abstract}

Methods: 34 cases of soft tissue defects of the volar thumb finger were included in the study, divided into three groups, in each group a flap was used for reconstruction of soft tissue defects of the volar thumb.

Results: volar palmar advancement flap is performed with no donor site morbidity, is good in terms of sensation and cosmetic results, should not be used for defects more than $1.5 \mathrm{~cm}$, heterodigital neurovascular flap is excellent in terms of restoration of sensation, function and cosmetic appearance., First dorsal metacarpal artery flap is the worst in terms of cosmetic results and sensation.
Volume 3 Issue 3 - 2017

\author{
Wael Naeem Thabet,' Joseph Naiem Sabet \\ Aziz $^{2}$ \\ 'Department of General Surgery, Cairo University Hospital, \\ Egypt \\ ${ }^{2}$ Department of Anatomy, Cairo University, Egypt
}

Correspondence: Joseph Naiem Sabet Aziz, Department of General Surgery, Cairo University Hospital, Egypt, Email jo_anatomy@yahoo.co.uk

Received: March II, 2016 | Published: March 17, 2017

Keywords: local flaps reconstructions, thumb defects, sensation, metacarpal

\section{Introduction}

The thumb plays an important role in hand function. Daily tasks involving pinch, grip, grasp and precise handling are more easily accomplished with an opposable thumb, Loss of thumb diminish much the hand abilities and function. ${ }^{1}$ Soft tissue injuries of the hand frequently require flap coverage, either to preserve structures or even to facilitate later reconstruction. ${ }^{2}$ The absence of the thumb, whether due to failure of formation or loss from trauma, significantly impairs hand function. ${ }^{3,4}$ The goals of thumb reconstruction are sensate and non tender thumb tip, stability of thumb joints, adequate length to resist the forces of the fingers, correct posture and postioning of the thumb with a wide adductor space. ${ }^{5}$

An ideal reconstruction of the thumb 'would replace like with like', restoring both function and appearance (Figures 1A-1E). Opposition, the hallmark of 'thumbness', necessitates length, stability, strength and mobility, exactly how important each of these factors becomes will vary depending on the needs of the patient, the jeweler who seeks sensibility and mobility may require different management than he manual laborer who may sacrifice mobility for stability and power. ${ }^{6,7}$

\section{Aim of the study}

To get an ideal reconstruction technique by comparing between different types of local flaps which are volar palmar advancement flap, heterodigital neurovascular island flap and first dorsal metacarpal artery flap for soft tissue reconstruction of volar thumb defects.

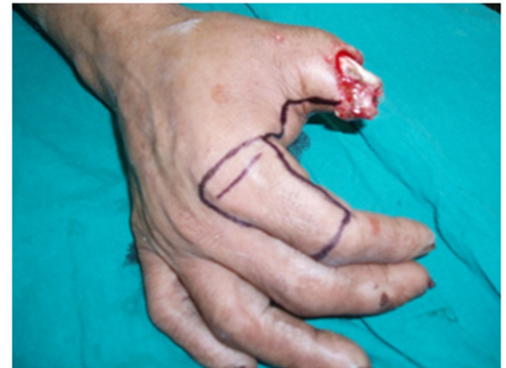

Figure I Preoperative view of a male patient, 33years showing the design of the first dorsal metacarpal artery flap.

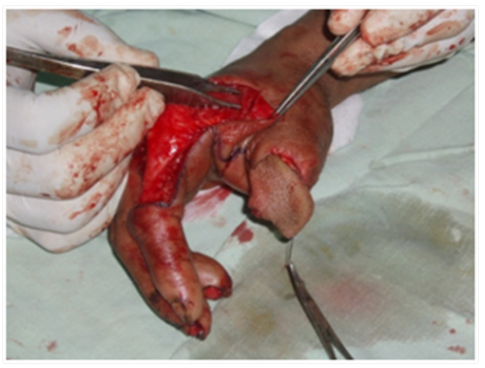

Figure IA Flap inset. 


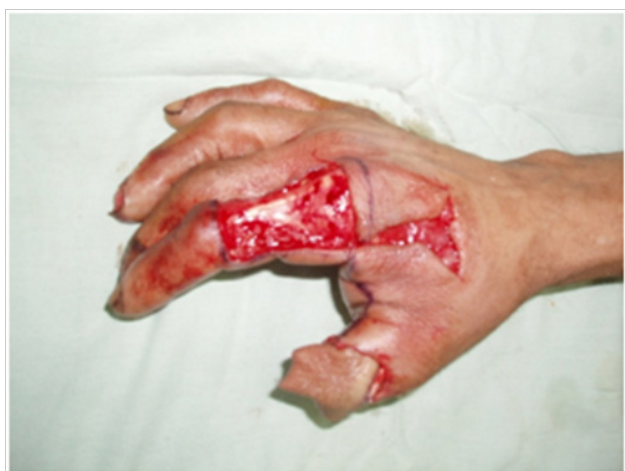

Figure IB Recipient site coverage.

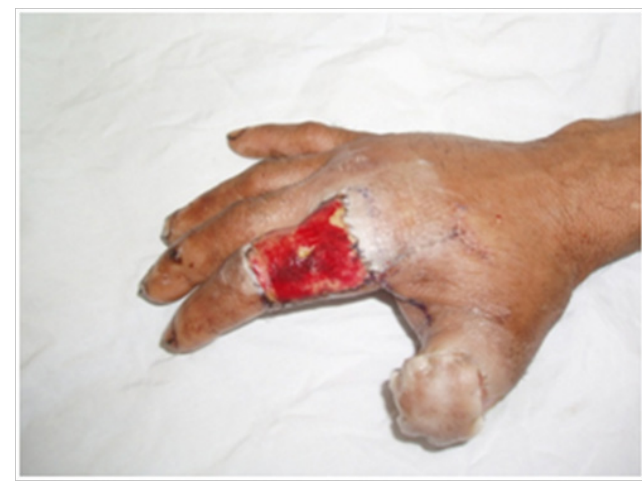

Figure IC Flap fixed to the defect.

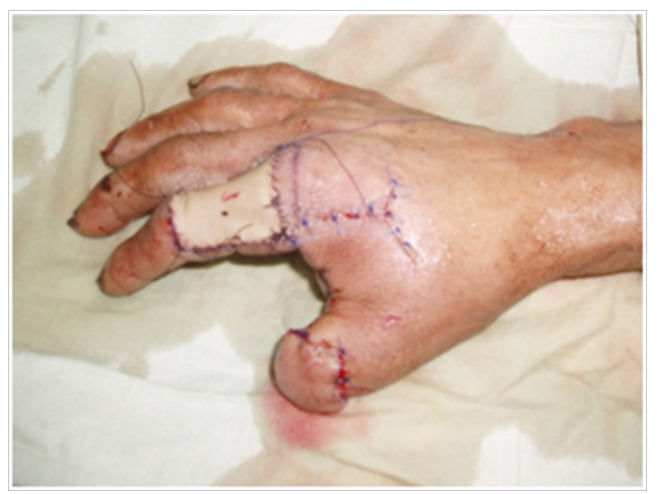

Figure ID Full thickness skin graft for donor site.

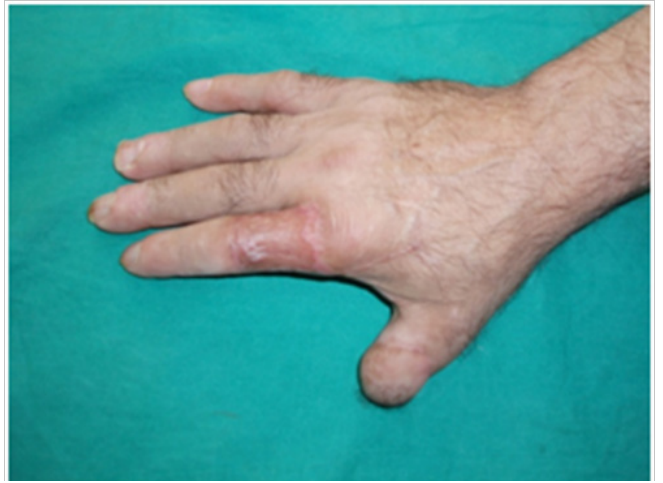

Figure IE Final appearance of the flap and the skin graft.

\section{Patients and methods}

The present work included 34 patients, 33 males and one female, with soft tissue defects of the thumb finger that presented and managed at Kasr Al-Aini Hospital (Cairo University) department of plastic surgery, between 2010-2012. All cases in this study were acute post-traumatic patients. Cases were divided into three groups, group A included 12 cases where volar palmar advancement flap was performed, group B included 8 cases where heterodigital neurovascular island flap was performed and group $\mathrm{C}$ included 14 cases where first dorsal metacarpal artery flap was performed. Mean surface area of soft tissue defects was $2.25 \mathrm{~cm}^{2}$, the mean follow up period was 2 months. In our study the surgical technique used to perform volar thumb advancement skin flap (Moberg Flap) included performing Longitudinal mid-lateral (mid-axial) incisions on each side (radial and ulnar sides) of the thumb. The volar skin with its subcutaneous tissue and both neurovascular bundles were elevated from the underlying tendon sheath of the flexor pollicis longus, and the flap was dissected and raised at the level of the flexor tendon sheath from distal to proximal. Advancement of the flap was obtained primarily by interphalangeal joint flexion, flexion of the inter-phalangeal joint will permit the flap to be advanced up to $1.5 \mathrm{~cm}$, if it is elevated to the flexion crease of the MP joint, the flap was advanced distally and sutured into the nail or the nail bed.

Postoperatively a dorsal plaster splint was applied, immobilizing the wrist in neutral position and the thumb in slight flexion, to relieve any tension on the skin sutures. Active thumb flexion was instituted immediately postoperatively and active extension exercises encouraged when the splint was discontinued in 10days. The surgical technique used to perform neurovascular (Littler) Flap included isolation of the ulnar border of the tip of the middle finger on its neurovascular bundle, dissection up into the palm, it was then tunneled for thumb reconstruction. The skin island was outlined on the ulnar aspect of the middle finger; an incision was then made along the ulnar lateral border of the finger and continued into the palm in a zigzag fashion. The dissection was performed under loupe magnification and tourniquet control, the digital artery, vein and nerve were easily identified in the finger, the skin island isolated and dissected off the underlying tissue, the flap was elevated from distal to proximal.

In the web space between the middle finger and ring finger, the arterial branch of the common digital vessel to the radial side of the ring finger was isolated, divided and ligated. The common digital nerve was splitted so as to preserve the innervation to the radial side of the ring finger, while keeping the branch to the ulnar side of the middle finger with the vascular pedicle during this proximal dissection. A tunnel was then made to the tip of the thumb and the neurovascular flap pulled through, inset and sutured without tension. The donor site was closed using a skin graft. Surgical technique used for performing dorsal metacarpal artery flap (Kite Flap) included a flap of skin from the dorsum of the proximal phalanx of the index finger based on the dorsal metacarpal artery dissected to reach and cover defects on the volar aspect of the thumb. Before starting the operation, the pulsation of the radial artery was checked against the second metacarpal bones at the angle between the tendon of extensor pollicus longus and the bone.

The flap was elevated in a distal to proximal fashion without prior pedicle dissection. Skin island on the proximal phalanx of the index finger was incised and elevated, leaving the well defined extensor paratenon intact, at the level of the metacarpal neck, a perforator was 
always present, this was coagulated or ligated. A zig zag or lazy $\mathrm{S}$ fashion incision was extended along the ulnar half of the first dorsal interosseous muscle to identify the first dorsal metacarpal artery and its associated vena comitants, the dissection included the ulnar half of the deep fascia over the dorsal surface of the first dorsal interosseous muscle to be included in the flap (Figure 2) (Figure 2A). The flap was then rotated to reach defects on the volar aspect of the thumb. In order to avoid first web space contracture, the skin over this space should not be included in the skin island. Pedicle dissection was done under loupe magnification, starting with elevation of thin skin flaps on both sides of the zig-zag or lazy $\mathrm{S}$ incision.

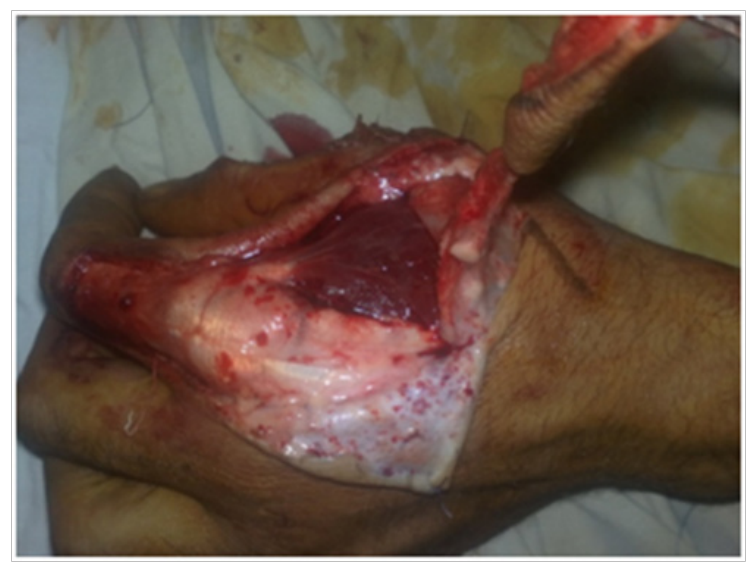

Figure 2 First dorsal interosseous flap elevation, the deep fascia over the first dorsal interosseous muscle is included in the flap.

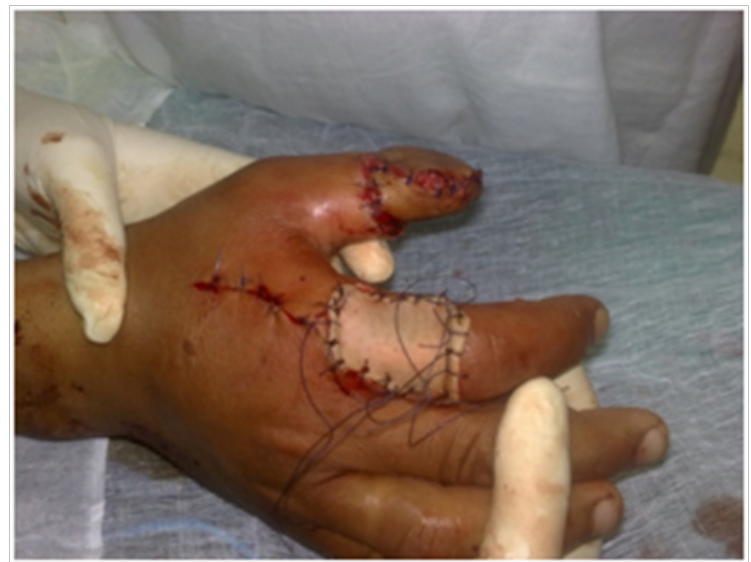

Figure 2A Postoperative photo.

Among the visible superficial dorsal veins, the ones that have about the same course with the pedicle were always preserved and incorporated to the pedicle with a cuff of superficial subcutaneous layer, inclusion of at least one or more superficial dorsal veins in the pedicle would increase venous outflow and help avoid postoperative flap congestion. The first dorsal metacarpal artery and associated venae comitants were located deep in the first dorsal web space and adherent to the ulnar half of dorsal surface of the first dorsal interosseous muscle, during the radial to ulnar elevation of the deep muscle fascia across the ulnar half of the muscle, the first dorsal metacarpal artery readily comes into vision. The periosteum at the dorsal radial edge of the second metacarpal bone was representing the deep ulnar limit of pedicle elevation and dissection in ulnar direction was stopped when this was reached. The pedicle included the interosseous fascia with the three branches of the first dorsal metacarpal artery, the superficial veins, the superficial radial nerve and its accompanying artery. The harvested island flap was tunneled to the defect. Split thickness skin graft was used to close the donor defect.

\section{Results}

Volar palmar advancement flap was done in twelve cases (35.3\%), heterodigital neurovascular flap in eight cases $(23.5 \%)$ and first dorsal metacarpal artery flap in fourteen cases $(41.2 \%)$. No complications were observed in twenty one cases. Nine cases of volar palmar advancement flap, five cases of heterodigital neurovascular flap and twelve cases of first dorsal metacarpal flap, were performed without minor or major complications (Figure 3) (Figure 3A) (Figure 3B). Delayed wound healing was observed in 3 cases of volar palmar advancement flap. Partial flap loss in one case of heterodigital flap and one case of first dorsal metacarpal flap, complete flap loss did not happen in any case, donor site morbidity occurred in one case of heterodigital neurovascular flap (12.5\%) in the form of numbness at the donor site, and in one case of first dorsal metacarpal flap (7\%) in the form of stiffness in proximal inter-phalangeal joint of the donor digit. Cosmetic disfigurement occurred in volar palmar advancement flap in one case $(8.3 \%)$ and in first dorsal metacarpal flap in two cases $(14.3 \%)$ and in one case $(12.5 \%)$ of heterodigital neurovascular flap. Assessment of outcome of different types of flaps used in reconstruction was done according to the following criteria: safety coverage, restoration of sensation and function, cosmetic appearance and donor site closure (Table 1) (Figure 4) (Figure 4A) (Figure 4B).

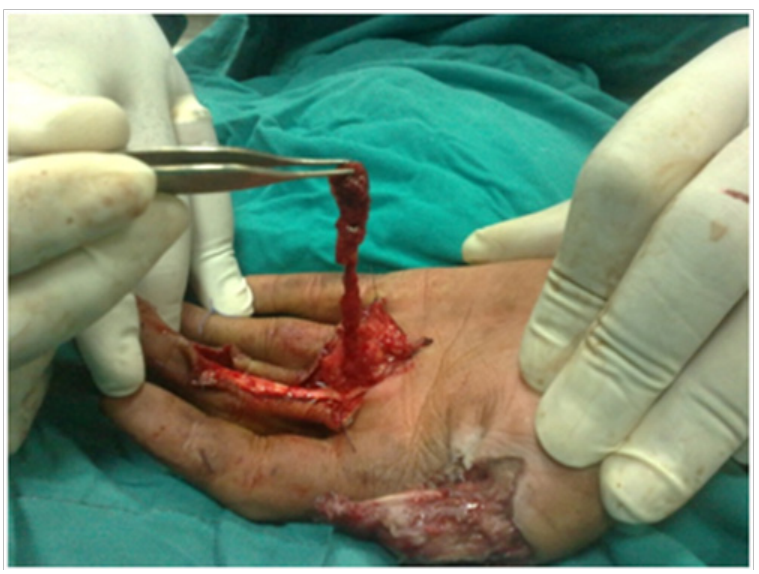

Figure 3 Heterodigital neurovascular island flap elevation.

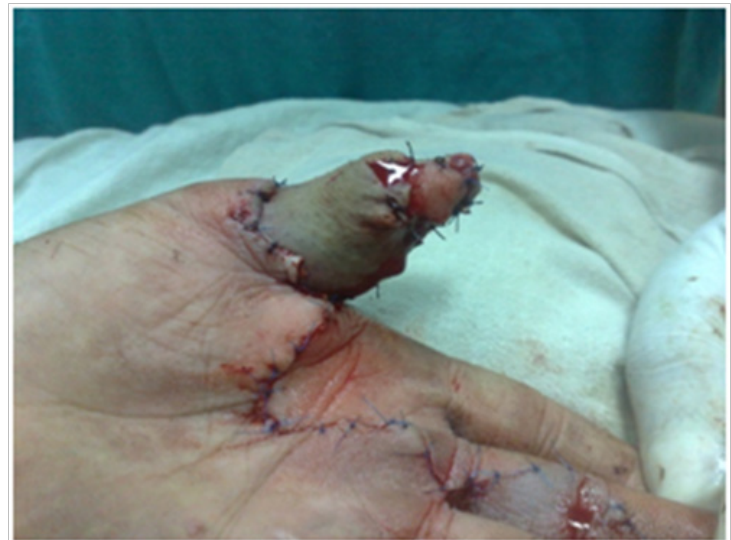

Figure 3A Flap inset. 


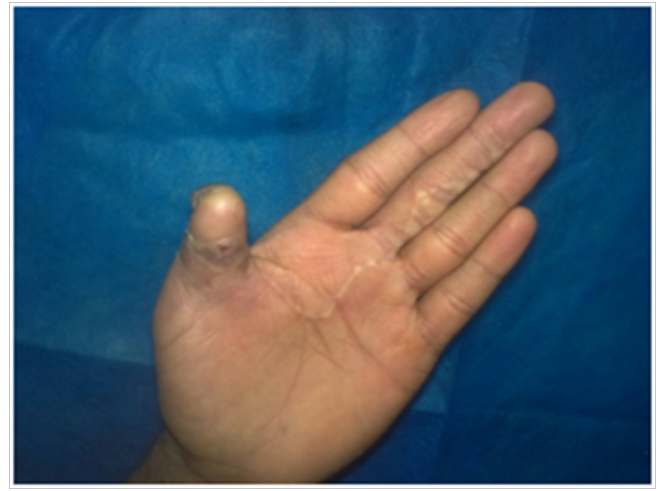

Figure 3b Final appearance of the same patient.

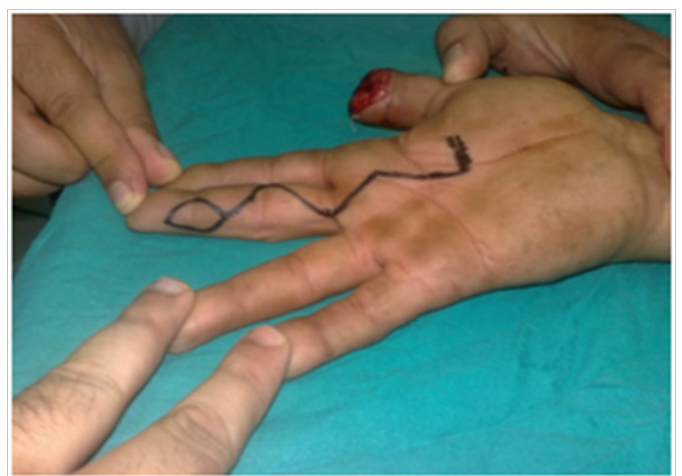

Figure 4 Heterodigital neurovascular flap design.

Table I Clinical and biochemical variables of individuals with overweight-obesity

\begin{tabular}{llllll}
\hline Flap type & $\begin{array}{l}\text { Safety } \\
\text { coverage }\end{array}$ & $\begin{array}{l}\text { Restoration of } \\
\text { sensation }\end{array}$ & $\begin{array}{l}\text { Restoration of } \\
\text { function }\end{array}$ & $\begin{array}{l}\text { Cosmetic } \\
\text { result }\end{array}$ & $\begin{array}{l}\text { Donor } \\
\text { morbidity }\end{array}$ \\
\hline Volar palmar & $75 \%$ & $88.80 \%$ & $83.30 \%$ & $91.70 \%$ & $0 \%$ \\
Heterodigital & $100 \%$ & $100 \%$ & $100 \%$ & $87.50 \%$ & $12.50 \%$ \\
Dorsal metacarpal & $92.90 \%$ & $64.30 \%$ & $85.70 \%$ & $85.70 \%$ & $7.10 \%$ \\
\hline
\end{tabular}

$\mathrm{SD}$, standard deviation; $\mathrm{BMI}$, body mass index; WC, waist circumference; $\mathrm{AC}$, abdominal circumference; $\mathrm{HC}$, hip circumference; RER, respiratory exchange ratio; HR, hear rate

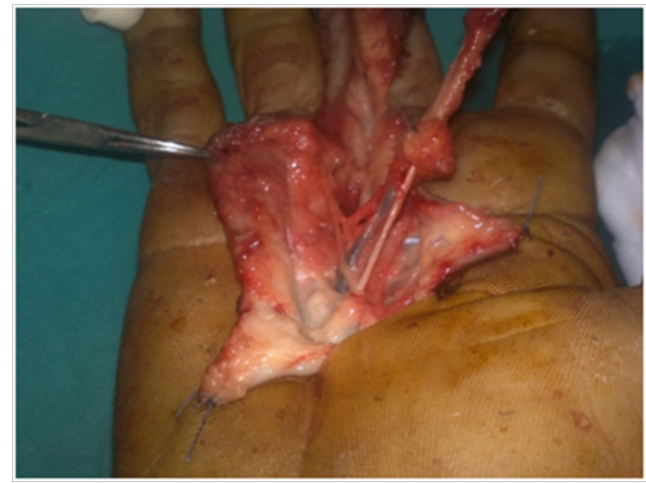

Figure 4A Flap elevation in the same patient.

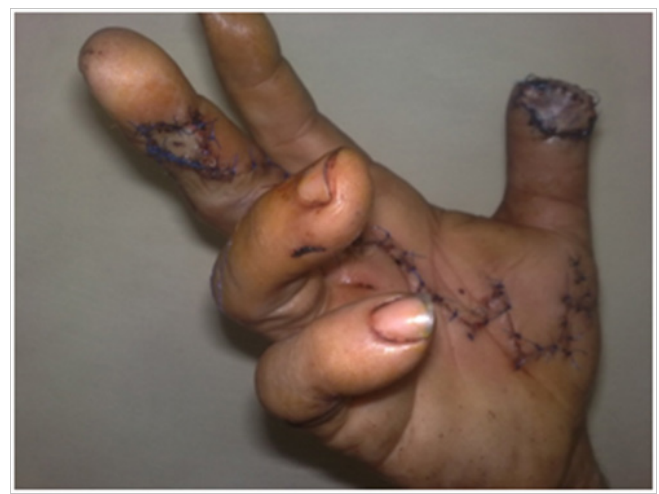

Figure 4B Flap inset.

\section{Discussion}

Defects of the thumb usually occur because of failure of closure of post-traumatic wounds. ${ }^{8}$ Soft tissue defects of the thumb frequently require flap coverage (Figure 5). ${ }^{2}$ The working surface of the thumb is best replaced with tissues that are glabrous as it is hairless, has fine sensibility and good pseudo motor function for tactile gnosis.
When possible, tissue should be from the same hand or the thumb itself and transferred as a local flap. Hallock ${ }^{9}$ mentioned that the use of local flaps for thumb reconstruction avoids the complexity of the microsurgical tissue transfer, allows earlier mobilization of the hand and digit than distal flaps. Borbly ${ }^{10}$ mentioned the following advantages of the axial pattern flaps over the random pattern flaps; wider arc of rotation, greater dimensions, more bacterial resistance, no need for a delay procedure and have a known vascular pedicle allowing for distal replacement so it can be used for areas away from the zone of injury. The identification of the vascular pedicles offered tremendous possibilities for wound coverage and defect reconstruction. ${ }^{11}$

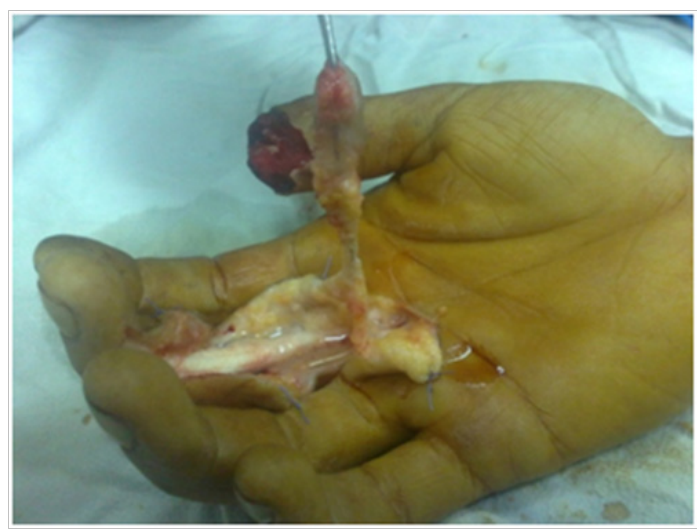

Figure 5 Heterodigital neurovascular flap elevation.

The Littler's flap (Fasciocutaneous heterodigital island neurovascular flap) was used in eight cases. The flap was reliable, with good vascular and sensory supply, and provided thumb reconstruction in a single stage. This meets what Braun, ${ }^{12}$ mentioned. This flap offered good results in terms of cosmetic appearance due to skin match and excellent results in terms of restoration of sensation as the digital nerve was preserved in the flap, on the other hand, the flap dissection required longer duration than the other flaps. The first dorsal metacarpal artery flap was done in fourteen cases. The 
flap was reliable, with good vascular supply, and provided thumb reconstruction in a single stage. Postoperatively, one partial flap loss occurred, another patient had stiffness in the proximal interphalangeal joint of the index (donor finger), good cosmetic results were achieved in $85.7 \%$ of the cases, the skin at the index finger dorsum didn't match well with the skin at the volar thumb surface (Figure 6) (Figure 6A) (Figure 6B).

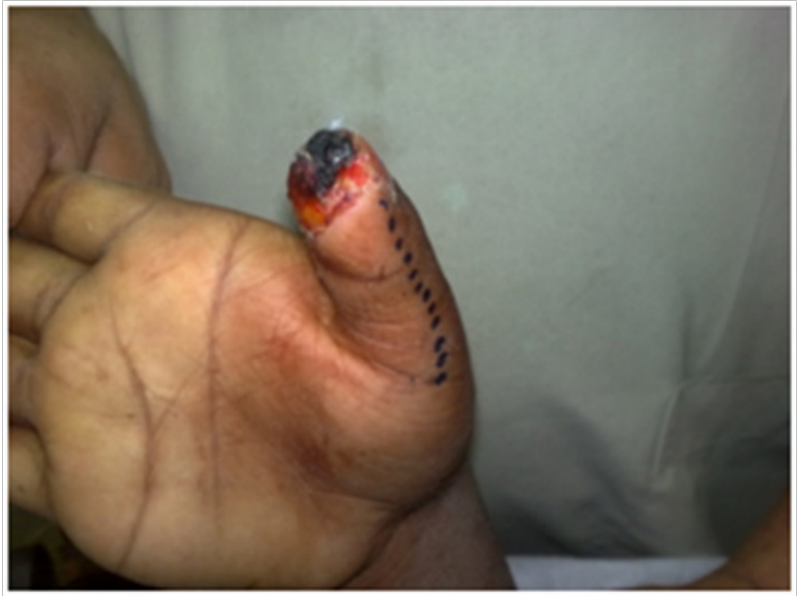

Figure 6 Volar palmar flap design.

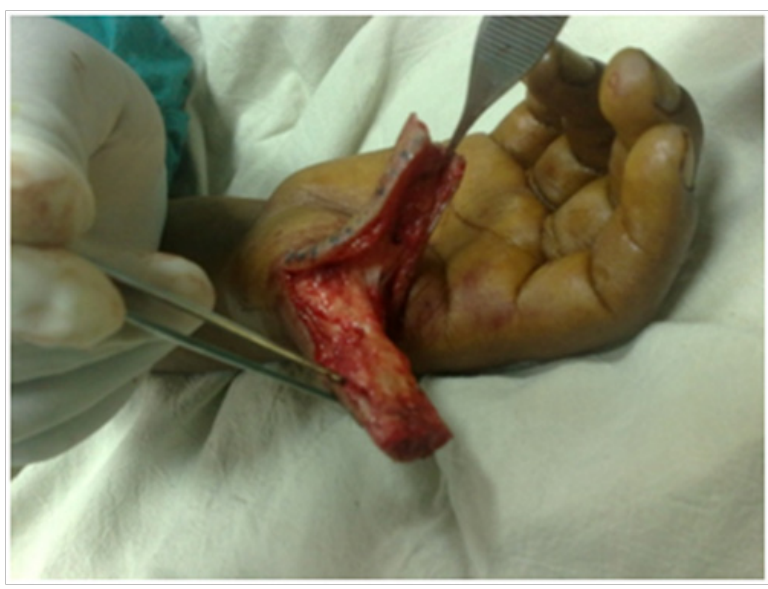

Figure 6A Volar palmar flap elevation.

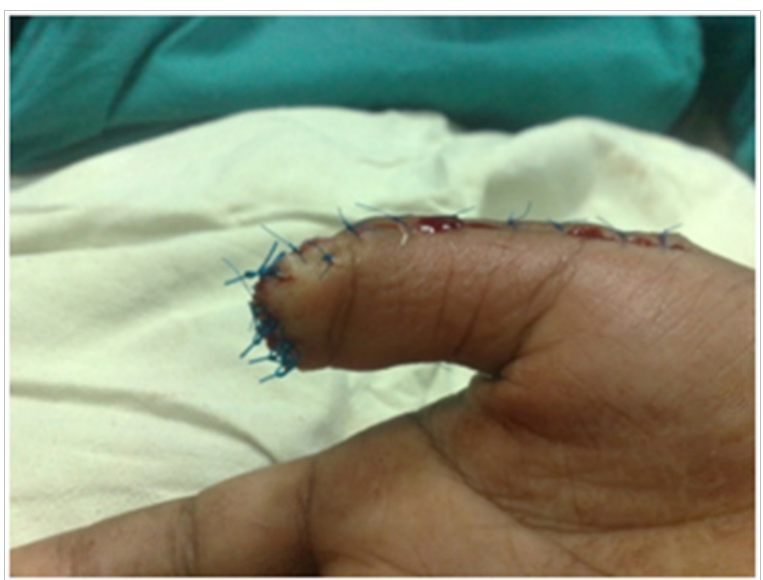

Figure 6B Volar palmar flap inset.
The mean result of the two point discrimination test in cases who performed Littler's flap, was $4 \mathrm{~mm}$, while the mean result of this test in cases who performed first dorsal metacarpal flap, was $8 \mathrm{~mm}$, hence, Litter's flap was found to be better than first dorsal metacarpal flap as regard to sensation, probably Littler's flap was better in terms of sensation because the flap contains a well defined sensory nerve that is the digital nerve, on the other hand, the first dorsal metacarpal artery flap has poor nerve supply as it contains the ramifications of the superficial radial nerve. Volar palmar advancement flap was done in twelve cases (Figure 7) (Figures 7A-7C). The flap was reliable, with good vascular and sensory supply, and provided thumb reconstruction with well padded skin, free from tender scars in a single stage with no donor site morbidity, this flap was found to be sufficient to cover no more than $1.5 \mathrm{~cm}$ of the volar surface of the thumb, and otherwise a flexion deformity may occur (Figure 8) (Figure 9).

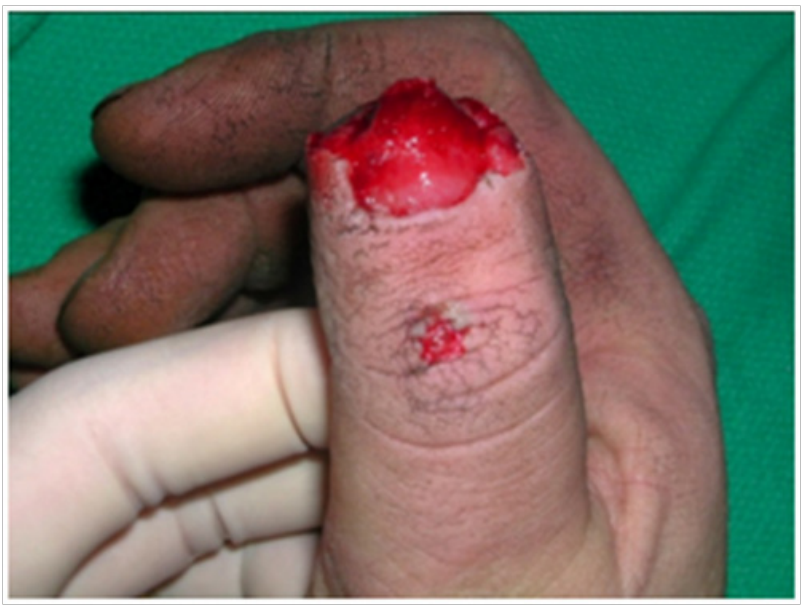

Figure $7 \mathrm{~A}$ case with volar thumb soft tissue defect.

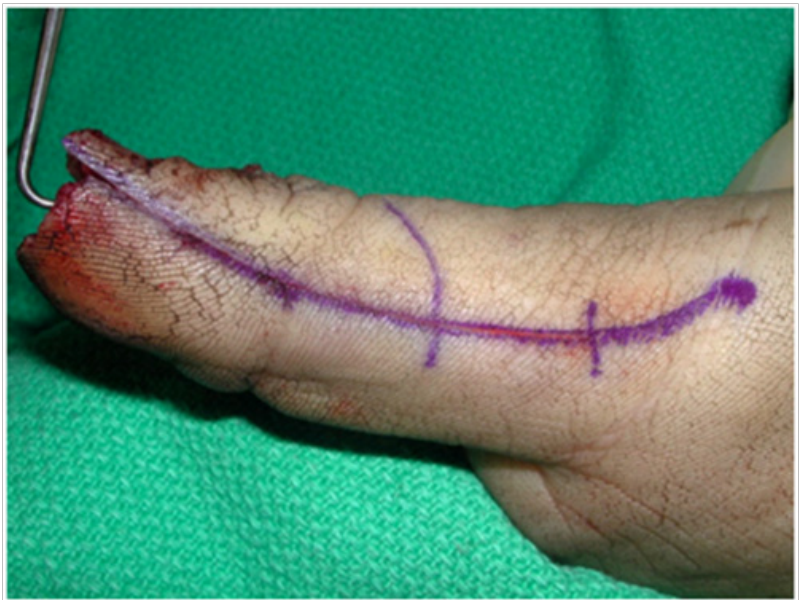

Figure 7A Volar palmar flap design in the same patient.

It may be concluded that local flaps could be efficiently used to manage cases of soft tissue defects at the volar aspect of the thumb. Inspite of the versatility of the recent micro-vascular reconstructive techniques, which proved to be satisfactory for the surgeon and the patient, the simpler and may be the older techniques, must remain in mind, because it is the commonest solution in many cases. It is the surgeon's responsibility to be imaginative and wise enough to secure the best solution for the patient. ${ }^{13}$ 


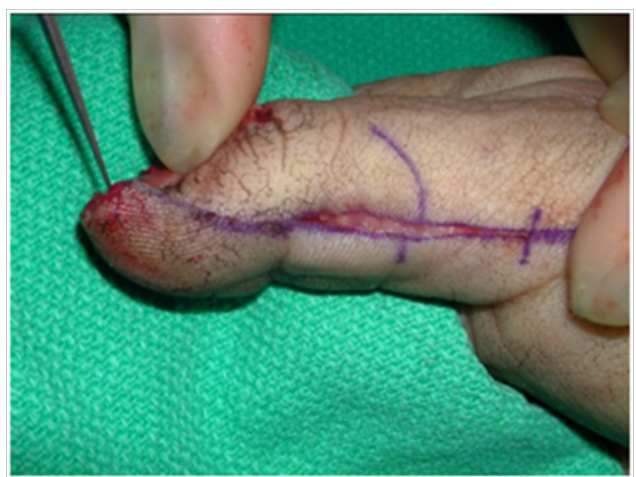

Figure 7B Flap advancement to thumb tip.

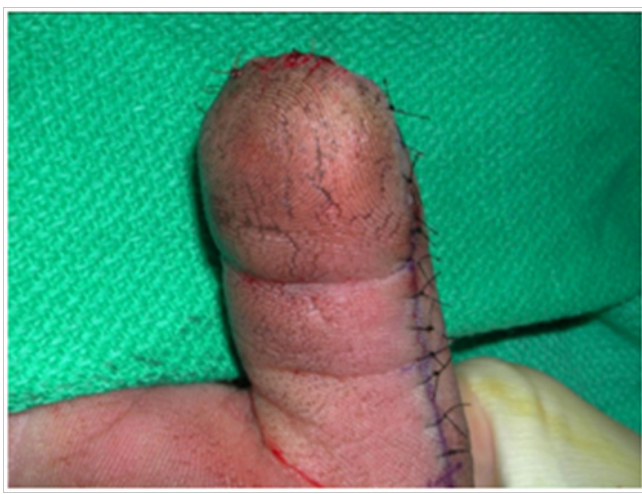

Figure 7C Flap covering the defect.
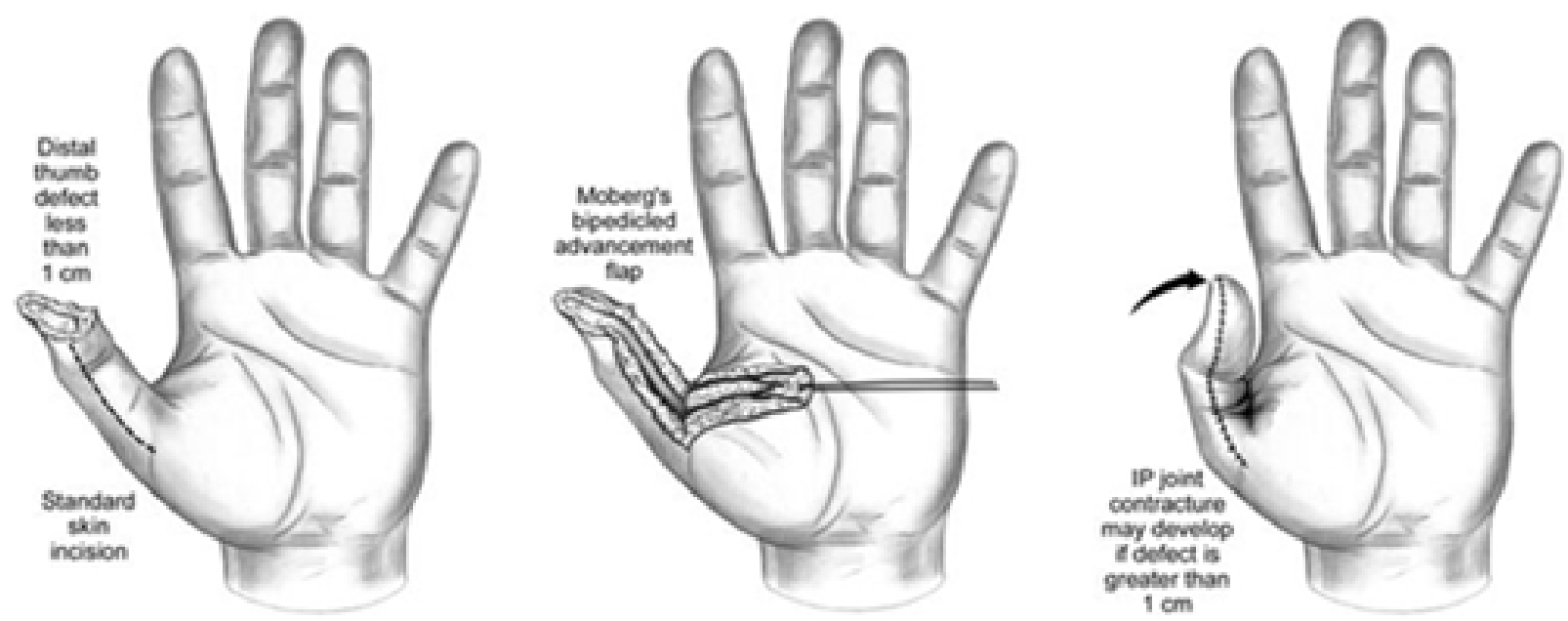

Figure 8 Diagrammatic presentation of the local flap for covering the thumb defect.

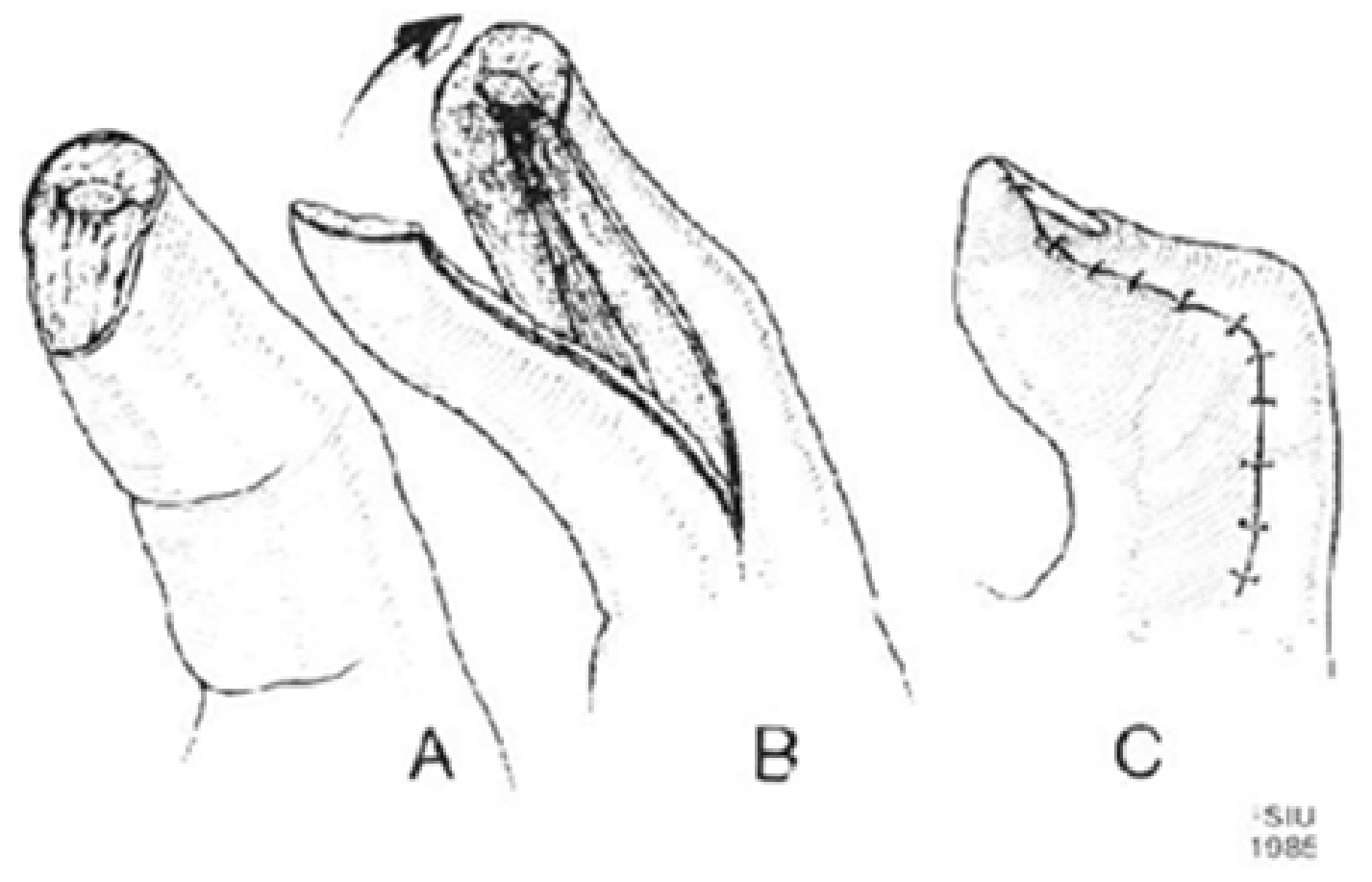

Figure 9 Diagram of a Moberg volar advancement flap being used for a thumb tip defect.

Citation: Thabet WN, Aziz JNS. A comparative study between different types of local flaps used for soft tissue reconstruction of volar thumb defects. MOJ Anat Physiol. 2017;3(3):84-90. DOI: 10.15406/mojap.2017.03.00092 


\section{Acknowledgements}

None.

\section{Conflict of interest}

Author declares that there is no conflict of interest.

\section{References}

1. Bueno RA, Wilhelmi BJ. Thumb reconstruction. 2007.

2. Soutar D, Tanner N. The radial forearm flap in the management of soft tissue injuries of the hand. Br J Plast Surg. 1984;37(1):18-26.

3. Livingston CK, Ruis-Razura A, Cohen BE. Guidelines for a successful microsurgery training center and research fellowship. Plast Reconstr Surg. 1999;104(5):1555-1558.

4. Lee A, Salyapongse N. Thumb reconstruction. Green's Operative Hand Surgery. 2005:1865-1866.

5. Hietmann C, Levin LS. Alternatives to thumb replantation. Plast Reconstr Surg. 2002;110(6):1492-1503.
6. Manketlow RT, Zuker RM, Mckee NH. Functioning free muscle transplantation. J Hand Surg. 1984;9A(1):32-39.

7. Vikki SK. Distraction and microvascular epiphysis transfer for radial club hand. J Hand Surg Br. 1998;23(4):445-452.

8. Davalbhakata A, Niranjan N. Fasciocutaneous flaps based on fascial feeding vessels for defect in the periolecranon area. Br J Plast Surg. 1999;52(1):60-63.

9. Hallok G. The local random Fasciocutaneous flaps for upper extremity coverage. J Hand Surg Am. 1992;17(1):93-101.

10. Borbly L. Axial and random skin flaps. Acta Chir Hung. 1986;27(3):185-192.

11. Mathes S, Nahai. Reconstructive Surgery: principles, anatomy and technique. 1st ed. New York: Churchill Livingstone; 1997.

12. Braun J. A new island flap transfer from the dorsum of the index to the thumb. Plast Reconstr Surg. 1979;63(3):344-349.

13. Katsaros J. Indications of soft tissue free flap transfer to the upper limb and the role of alternative procedures. Hand Clinics. 1992;8:479. 